\section{Tschebychev Approximation to the Equation of Thermogravimetric Data}

THE use of approximate expressions rather than tabulations for non-elementary functions, such as the exponential integral, is often predicated on the simplicity of computation and the attainable accuracy. Since approximations receive quite wide usage, it is generally recognized that many expressions of varying degrees of complexity and precision are available. However, it is not so widely realized by workers not directly concerned with numerical analysis that techniques exist which may be used to obtain maximurn accuracy from given functional forms by varying appropriate constants within the expressions. Procedures which best achieve the desired result require that the constants be selected such that the maximum deviation of the approximant from the exact function be minimized over the selected range of the independent variable. This is a statement of the Tschebychev criterion (for a brief introduction see ref. 1 ). It is the purpose of this communication to present a Tschebychev approximation to the function $p(x)$, given by Doyle $^{2}$ as :

$$
p(x)=\mathrm{e}^{-x} x^{-1}-\int_{x}^{\infty} \mathrm{e}^{-\tau} \tau^{-1} \mathrm{~d} \tau=\mathrm{e}^{-x} x^{-1}-E_{1}(x)
$$

which occurs in the equation of the thermogravimetric data plot.

Doyle $^{2}$ indicates that the use of truncations of the Schlömilch expansion yields:

$$
p(x) \sim \mathrm{f}(A, x)=(x+A)^{-1} x^{-1} \mathrm{e}^{-x}, x \geqslant 10
$$

where $A$ is 1 for the two-term expansion, and 2 for the three-term expansion.

The functional form, sufficiently simple for computation, possesses maximum relative errors of $-7 \cdot 2$ per cent and $+1 \cdot 3$ per cent, respectively, for $x \geqslant 10$. It is clear that some different selection of $A$ should permit a higher accuracy with no increase in complexity.

To accomplish the fit, we seek to minimize (relative deviation):

$$
\max _{x \geqslant 10}\left|\frac{\Theta^{-x} x^{-1}-E_{1}(x)-(x+A)^{-1} x^{-1} \theta^{-x}}{(x+A)^{-1} x^{-1} \Theta^{-x}}\right|
$$

that is, select $A$ such that:

$$
\delta=\min _{A} \max _{x \geqslant 10}\left|1-x(x+A) \mathrm{e}^{x} E_{1}(x)\right|
$$

The normal error curve for a problem of this type will possess two extrema, $\pm \delta$, the location of which may be obtained by application of the Remes second algorithm ${ }^{3,4}$. The algorithm is effected by: (1) assuming a set of abscissee for which extrema might be expected (for example, $\left.x_{1}=10, x_{2}=50\right)$; (2) solving for $A$ and $\delta$ (deviation of $+\delta$ at $x_{1},-\delta$ at $x_{2}$ ); (3) determining the abscissae of the true extrema of the resultant error curve; (4) using these as elements for a continuing iteration. This can easily be done for the foregoing function yielding:

$$
\begin{aligned}
& x_{1}=10, x_{2} \sim 27 \\
& A=1.8768 \\
& \delta=2.0 \times 10^{-3}=0.2 \text { per cent, } x \geqslant 10
\end{aligned}
$$

It should be noted that an improvement of an order of magnitude is obtained over the relative error of the threeterm Schlömilch expansion.

\begin{tabular}{ccc} 
& \multicolumn{2}{c}{ Table 1. $p(x) / \mathrm{f}(A, x)$} \\
$x$ & $\begin{array}{c}\text { Schlömilch three-term } \\
(\boldsymbol{A}=2)\end{array}$ & $\begin{array}{c}\text { Tschebychev fit } \\
(\boldsymbol{A}=1.8768)\end{array}$ \\
10 & 1.013 & 1.0020 \\
15 & 1.007 & 0.9990 \\
20 & 1.003 & 0.9982 \\
25 & 1.002 & 0.9980 \\
30 & 1.001 & 0.9980 \\
35 & 1.000 & 0.9981 \\
40 & 1.000 & 0.9982 \\
45 & 1.000 & 0.9982 \\
50 & 1.000 & 0.9983 \\
100 & 1.000 & 0.9990 \\
200 & 1.000 & 0.9994
\end{tabular}

I thank Dr. J. Ross Macdonald for directing my attention to this application and Dr. C. D. Doyle for his advice.

Eric L. JONFs

Texas Instruments, Incorporated, Dallas, Texas.

${ }^{1}$ Hamming, R. W., Numerical Methods for Scientists and Engineers (McGrawHill Book Co. New York, 1962).

${ }^{2}$ Doyle, C. D., Nature, $207,290(1965)$

${ }^{3}$ Remes, E., C.R. Acad. Sci., Paris, 199, 337 (1934).

'Murnaghan, F. D., and Wrench, J. W., Math. Tables Aids Comput., 13, $185(1959)$.

\section{Calculations with Two Nearly Equal Quantities}

Dr. WORRELL ${ }^{1}$ has suggested that, when a small signal is to be detected against a large background, more reliable results can be gained by using ratios of observations than by using differences. For example, in a counting experi. ment where $S$ events in a standard interval are to be detected against a background of $B$ events, he shows that the relative error in $S=(S+B)-B$ is:

$$
e_{s}=\sqrt{\frac{S+2 B}{S^{2}}}
$$

while the relative error in $R=(S+B) / B$ is:

for large values of $B$.

$$
e_{r}=\sqrt{\frac{S+2 B}{B(S+B)}}
$$

For small values of $S, e_{r}$ is clearly much smaller than $e_{s}$. While this result is indeed entirely reliable, its importance is not clear. If $S / B \rightarrow 0, e_{s}$ becomes large compared to I for any finite value of $B$, while

$$
e_{r} \rightarrow \sqrt{\frac{\overline{2}}{B}}
$$

which can be made as small as desired by using long counting times. Hence no indication is given that $S$ even exists, but $R$ can readily be found to high accuracy.

The drawback is that the value of $R$ so accurately found is $1 \cdot 0$..., which physically leads to the identical result that no indication at all is given that $S$ even exists.

The quantity of physical significance is, of course, not $R$ but $(R-1)$, since it is only the changes in $R$ from unity which can carry any information as to the existence or magnitude of $S$.

Now:

$$
R-1=\frac{B+S}{B}-1=\frac{(B+S)-B}{B}
$$

The relative error in this is:

$$
\sqrt{\frac{S+2 B}{S^{2}}}
$$

or exactly $e_{s}$, again for large values of $B$. The gain in reliability of the value of $R$ is thus of no practical interest.

\section{J. H. Fremlin}

Department of Physics,

University of Birmingham.

${ }^{1}$ Worrell, F. 'T., Nature, 207, 621 (1965).

WhILE most of the points in the foregoing communication are correct, I believe that Fremlin has misinterpreted my remarks. There seem to have been two points in my letter that were not clearly made. One was the nature of the experimental situation in which the ratio calculation was found to be useful; the other was my statement that "information can be extracted with greater reliability by taking the ratios rather than the differences". This state- 